\title{
On the minimum number of states for switchable matching networks
}

\author{
Ettore Lorenzo Firrao, Anne-Johan Annema, Frank E. van Vliet and Bram Nauta
}

\begin{abstract}
The impedance of an antenna changes heavily with changing EM environments, while RF power amplifiers (PAs) are optimized for driving a well-defined load impedance. As a solution, switchable matching networks are used in automatic antenna tuners to match the antenna impedance to (about) the desired PA load impedance. This paper presents a theoretical treaty of the minimum number of required states for switchable matching networks to achieve sufficient matching from a certain load VSWR to a sufficiently low input VSWR. First for an arbitrary passive lossless switchable matching network, the mathematical minimum required number of states as a function of the required input VSWR and of the required load VSWR is derived. Several variants are analyzed and benchmarked: single-stage one-ring configuration, single-stage two-ring configuration, two-stage one-ring configuration and threestage one-ring configuration showing that single-ring configurations are optimum. An extension towards the required number of states for lossy matching networks is also provided.
\end{abstract}

Index Terms - automatic antenna tuners, impedance matching, tunable matching networks, switchable matching networks.

\section{INTRODUCTION}

A ntenna impedances depend heavily on their EM environment [1][2] and hence may change significantly during real life operation. The antenna impedance $Z$ is usually expressed in terms of reflection coefficient $\Gamma_{Z}=\left(Z-Z_{0}\right) /\left(Z+Z_{0}\right)$, where $\mathrm{Z}_{0}$ is the characteristic impedance, or in terms of voltage standing wave ratio $\operatorname{VSWR}_{Z}=\left(1+\left|\Gamma_{Z}\right|\right) /\left(1-\left|\Gamma_{Z}\right|\right)$. For a typical antenna the VSWR can be up to 10:1 [1-5].

This changing antenna impedance causes serious design and performance challenges for the RF power amplifier (PA) driving the antenna due to the associated VSWR. Assuming a certain required lower bound on maximum transmit power, a varying load impedance for the PA (e.g. antenna impedance through a fixed matching network) requires robustness to both maximum voltage and maximum current levels well above those required when driving a nominal load impedance at the same power level [3]-[5]. Consequently, the PA then must be designed to operate properly for a wide load impedance range and hence the PA is necessarily overly robust at nominal conditions and consequently has significantly lower efficiency at nominal conditions. On top of this, matching losses due to non-nominal load impedances decrease the efficiency and radiated power significantly.

Automatic antenna tuners may be used as solution for this. In general, an automatic antenna tuner consists of impedance sensors, a control network and a tunable matching network [6]-[24]. The sensors are used to detect the impedance mismatch or impedance value; the control network controls the state or setting of the tunable matching network that performs the actual impedance tuning from its load impedance $\mathrm{Z}_{\text {load }}$ into its input impedance $\mathrm{Z}_{\text {in }}$.

The tunable matching network can be implemented with continuous tunable components or with switchable components. In case of continuous tunable components it is theoretically possible to achieve a perfect tuning at the input for a certain (limited) load impedance region. In case of ideal switchable components perfect impedance matching can be achieved for only a finite number of load impedances. This paper presents a mathematical estimation of the minimum required number of states for narrow band switchable matching networks to match a certain load impedance range to within a smaller input impedance range. This problem was implicitly addressed in [22]-[24] for specific implementations, without giving a general approach or mathematical derivation.

Section II presents a mathematical estimation of the minimum required number of states for a single stage switchable matching network assuming a resistive source driving a lossless switchable matching network terminated by a load impedance (e.g. the antenna). This derivation uses the mismatch efficiency [25] in order to keep the mathematical formalism as general as possible without referring to specific implementations: the only assumption is that the switchable matching network is passive and lossless; it can be reciprocal or non-reciprocal. Consequently, the results are valid for any (yet lossless) switchable single stage matching network. The resulting minimum required number of states can be used to derive a lower bound on hardware costs to implement switchable matching networks, and can be used to estimate the efficiency of specific hardware implementations in terms of overhead in states. 
Sections III to V extend the results of section II in a number of ways. Section III discusses the merits and limitations of multi stage matching networks: it shows that partitioning a matching network in multiple simpler matching networks can be very efficient in terms of minimum total required number of states to perform a certain impedance matching. Section IV briefly discusses the impact of using an arbitrary complex source impedance instead of a purely real source resistance, while an extension towards (general) lossy matching networks is discussed in section V. Section VI summarizes the findings.

\section{NEAR MINIMUM NUMBER OF STATES FOR LOSSLESS SWITCHABLE MATCHING NETWORKS}

This section presents a mathematical derivation of the minimum required number of states for lossless switchable matching networks at a single frequency. The matching network matches any load impedance $Z_{\text {load }}$ in a certain impedance range to impedances $Z_{i n}$ that are sufficiently close to the complex conjugate of the source impedance. In this section, a real source impedance $R_{\text {source }}$ is assumed.

In this paper, complex impedances $Z$ are mainly specified in terms of reflection coefficient $\Gamma_{Z}=\left(Z-Z_{0}\right) /\left(Z+Z_{0}\right)$. Some basic properties of this bilinear transformation between $Z$ and $\Gamma$ form the mathematical basis for the work in this paper. A first property is that the entire complex Z-plane is mapped to the entire complex $\Gamma$-plane with the point $\Gamma=1$ removed. Secondly, the bilinear transformation is holomorphic: it maps circles in the Z-plane into circles in the $\Gamma$-plane; non-overlapping circles in one plane are non-overlapping in the other plane [25, chapter 10].

The "number of states" denotes the actual number of different settings that the switchable matching network can have. Assuming for example a matching network that incorporates in total $N$ switched reactances, the number of states for that network equals $2^{N}$ of which a number may be coinciding, thereby effectively reducing the actual number of (unique) states. Assuming e.g. a slide-screw tuner that can be set in $N$ discrete settings, the number of states typically equals $N$. In this paper we however do not assume any specific implementation for the matching network.

In the current paper, the switchable matching network aims to match a load impedance $Z_{\text {load }}$ into a $Z_{\text {in }}$ that is sufficiently close to target impedance $R_{\text {source}}$, using a minimum number of required states. This "sufficiently close" means that the associated magnitude of the reflection coefficient $\Gamma_{\text {Zin }}$ is lower than a predefined value $\left|\Gamma_{\text {Zin }}\right|_{\max }$.

\section{A. Single state impedance matching}

The mismatch efficiency $M$ equals the ratio of actual power in the load $P_{\text {load }}$ and the maximum achievable power into the load $P_{\max }$ [25]. For a passive lossless matching network, see Fig 1 , at a single frequency this $M$ is a function of the complex source and load impedances seen at a cross section. At cross section A in Fig. 1 this translates into

$$
M=\frac{P_{\text {load }}}{P_{\max }}=\frac{\left(1-\left|\Gamma_{\text {Zsource }}\right|^{2}\right) \cdot\left(1-\left|\Gamma_{\text {Zin }}\right|^{2}\right)}{\left|1-\Gamma_{\text {Zsource }} \Gamma_{\text {Zin }}\right|^{2}},
$$

showing that maximum power transfer is achieved for $\Gamma_{\text {zsource }}=\Gamma_{\text {zin }}^{*}$.

The derivations in this paper are valid both for reciprocal and for non-reciprocal networks. Note that for lossless matching networks the $M$ is the same at cross section A and B in Fig. 1. For simplicity reasons we first assume a real source impedance $R_{\text {source }}$.

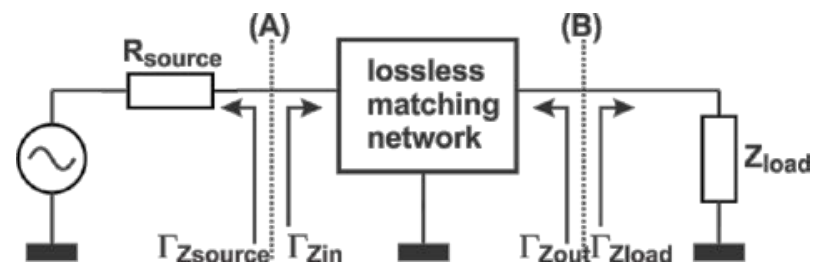

Fig. 1. Circuit topology and naming conventions in the derivation.

At a single frequency the impedance matching network can be designed to match a load impedance $Z_{\text {load,match }}$, with a reflection coefficient $\Gamma_{\text {Zload,match }}$, to the center of the Smith chart. For this perfect match, M=1 and $\Gamma_{\text {Zout }}=\Gamma_{\text {Zload,match }}^{*}$, as depicted in Fig. 2. 


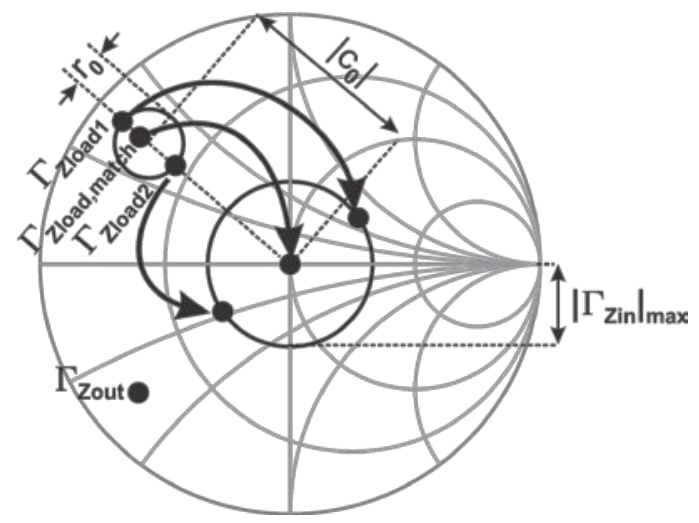

Fig. 2. Mapping of one load impedance circle onto an input impedance circle for a certain matching efficiency M.

For this matching network, a certain mismatch efficiency $\mathrm{M}<1$ corresponds to all $\Gamma_{\text {Zin }}$ lying on a circle centered on the center of the Smith chart having a radius $\left|\Gamma_{\text {Zin }}\right|_{\max }=\sqrt{1-M}$. Due to the bilinear properties of the matching networks, this circle for $\Gamma_{\text {Zin }}$ corresponds to a circle of $\Gamma_{\text {Zload }}$ values on the Smith chart. This latter circle encloses $\Gamma_{\text {zload,match }}$, but in general is not centered on $\Gamma_{\text {zload,match }}$ due to the compressive character of the Smith chart with larger distances towards its center point.

A description of this circle can be obtained by solving (1), for all $\left|\Gamma_{\text {zload }}\right|$ values at cross section B (see Fig. 1) for a given $M$. Under assumption that the resulting $\Gamma_{\text {zload }}$-circle does not encircle the center of the Smith chart, it can be proven that the center of the circle lies on the line through the center of the Smith chart and $\Gamma_{\text {zload,match }}$. Using the previously described matching network that matches $\Gamma_{\text {Zload,match }}$ onto to the center of the Smith chart, there are now two impedances $\Gamma_{\text {Zload1 }}$ and $\Gamma_{\text {Zload } 2}$ that lie on a line in the Smith chart crossing both the center point and crossing $\Gamma_{\text {zload,match }}$ that are matched onto impedances corresponding to some points on a circle around the origin with radius $\left|\Gamma_{\text {Zin }}\right|_{\max }$ that corresponds to the specific $M$, see Fig. 2 for a graphical representation. Solving (1) yields:

$$
\left|\Gamma_{\text {Zload } 1,2}\right|=\frac{\beta\left|\Gamma_{\text {Zout }}\right| \pm \sqrt{\left(\beta\left|\Gamma_{\text {Zout }}\right|\right)^{2}-(\beta-1)\left(1+\beta\left|\Gamma_{\text {Zout }}\right|^{2}\right)}}{1+\beta\left|\Gamma_{\text {Zout }}\right|^{2}}
$$

with $\beta=\frac{M}{1-\left|\Gamma_{\text {Zout }}\right|^{2}}$ and where $\Gamma_{\text {Zout }}=\Gamma_{\text {Zload,match }}^{*}$.

The center $\mathrm{c}_{0}$ and the radius $\mathrm{r}_{0}$ of the circle that is mapped by the matching network onto a circle around the origin with radius $\left|\Gamma_{\text {Zin }}\right|_{\text {max }}$ follow from (2):

$$
\begin{gathered}
c_{0}=\frac{\left|\Gamma_{\text {Zload } 1}\right|+\left|\Gamma_{\text {Zload } 2}\right|}{2}=\frac{M \Gamma_{\text {Zout }}^{*}}{\left|\Gamma_{\text {Zout }}\right|^{2}(M-1)+1}, \\
r_{0}=\frac{\left|\Gamma_{\text {Zload } 2}\right|-\left|\Gamma_{\text {Zload } 1}\right|}{2}=\frac{\left(1-\left|\Gamma_{\text {Zout }}\right|^{2}\right) \sqrt{1-M}}{\left|\Gamma_{\text {Zout }}\right|^{2}(M-1)+1} .
\end{gathered}
$$

\section{B. Single ring impedance matching}

Due to the bilinear nature of impedance transformations of switchable matching networks, circles described by (3) and (4) can be mapped onto circles centered at the origin of the Smith chart with radius $\left|\Gamma_{\text {Zin }}\right|_{\max }$. Using many suitably positioned circles a whole band can be constructed, directly outside the circles with radius $\left|\Gamma_{\text {Zin }}\right|_{\max }$ that can be mapped onto the center circle [22,23], see Fig. 3. 


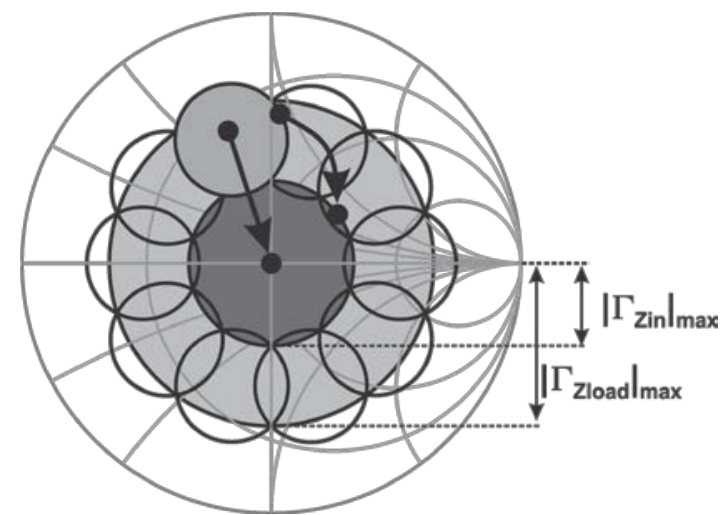

Fig. 3. Constructing a matchable area with radius $\left|\Gamma_{\text {Zload }}\right|_{\max }$ matchable onto a circle around the origin with radius $\left|\Gamma_{\text {Zin }}\right|$, using multiple (here 10+1) number of states for switchable matching networks.

Fig 3. illustrates such a matching network configuration with in total 11 states, having one center circle with sufficiently low $\left|\Gamma_{\text {Zin }}\right|$, and 10 circles around it that can individually be mapped onto the center circle, and in total forming the largest region matchable region described by $\left|\Gamma_{\text {Zload }}\right| \leq\left|\Gamma_{\text {Zload }}\right|_{\max }$. Impedance mapping of two distinct impedances in one of the outer circles onto the center circle is also depicted in Fig. 3.

It appears that analytically deriving the required number of matching network states as a function of $\left|\Gamma_{\text {Zin }}\right|_{\text {max }}$ and $\left|\Gamma_{\text {Zload }}\right|_{\max }$ is (at least) hard. However, it is possible to derive the region that can be matched to an impedance inside a circle with radius $\left|\Gamma_{\text {Zin }}\right|_{\max }$ as a function of the number of matching network states.

Using only one ring consisting of $\mathrm{N}_{1}$ circles (each circle corresponds to a single matching network state) around the center (see Fig. 3), the total circle that can be matched follows from using (3) and (4) in plain geometrical constructions to build a larger circle. This yields a $6^{\text {th }}$ order equation with four trivial or not-relevant solutions and one solution leading to a matched overall circle with radius (see appendix A):

$$
\begin{aligned}
& \left|\Gamma_{\text {Zload }}\right|_{\text {max }}=\left|\Gamma_{\text {Zin }}\right|_{\text {max }}+ \\
& \quad+2 r_{1} \cos \left(\frac{\pi}{N_{1}}+\arcsin \left(\frac{\left|\Gamma_{\text {Zin }}\right|_{\max } \sin \left(\frac{\pi}{N_{1}}\right)}{r_{1}}\right)\right),
\end{aligned}
$$

where

$$
r_{1}=\frac{\left(1-h_{1}^{2}\right)\left|\Gamma_{\text {Zin }}\right|_{\text {max }}}{1-h_{1}^{2}\left|\Gamma_{\text {Zin }}\right|_{\text {max }}^{2}}, h_{1}=\frac{2\left|\Gamma_{\text {Zin }}\right|_{\text {max }} \cos \left(\frac{\pi}{N_{1}}\right)}{1+\left|\Gamma_{\text {Zin }}\right|_{\text {max }}^{2}} .
$$

Boundary condition for this relation include having a real value for $\left|\Gamma_{\text {Zload }}\right|_{\max },\left|\Gamma_{\text {Zload }}\right|_{\text {max }}>\left|\Gamma_{\text {Zin }}\right|_{\max }$ and laying inside the Smith chart of the all circles (i.e. the regions that can be matched onto the center circle). If one or more of these boundary conditions are not satisfied, the parameter $\mathrm{N}_{1}$ is just too low to find a valid solution. Fig. 4 shows a the radius of the matchable area on the Smith chart $\left|\Gamma_{\text {zload }}\right|_{\max }$ as a function of the number of matching network states $N_{\text {tot }}=\left(N_{1}+1\right)$, for a number of $\left|\Gamma_{\text {Zin }}\right|_{\max }$-values. The “+ 1 ” is there because the circle centered at the center of the Smith chart must be included as state.

For low $N_{\text {tot }}$, the matched area does not form a circle larger than the center circle, hence the lower clipping of $\left|\Gamma_{\text {zload }}\right|_{\text {max }}$ on $\left|\Gamma_{\text {Zin }}\right|_{\max }$ in Fig. 4. With increasing $N_{\text {tot }}$ the radius of the matchable area increases and reaches (for $N_{\text {tot }} \rightarrow \infty$ ) the maximum radius asymptotically. Note that for clarity reasons, the graph in Fig. 4 shows continuous curves while $N_{\text {tot }}$ can only assume integer values. It follows from (5) and (6) that the maximum radius is:

$$
\left|\Gamma_{N \rightarrow \infty}\right|=\left|\Gamma_{\text {Zin }}\right|_{\max }\left(1+\frac{2\left(1-\left|\Gamma_{\text {Zin }}\right|_{\text {max }}^{2}\right)^{2}}{\left(1+\left|\Gamma_{\text {Zin }}\right|_{\text {max }}^{2}\right)^{2}-4\left|\Gamma_{\text {Zin }}\right|_{\text {max }}^{4}}\right) .
$$




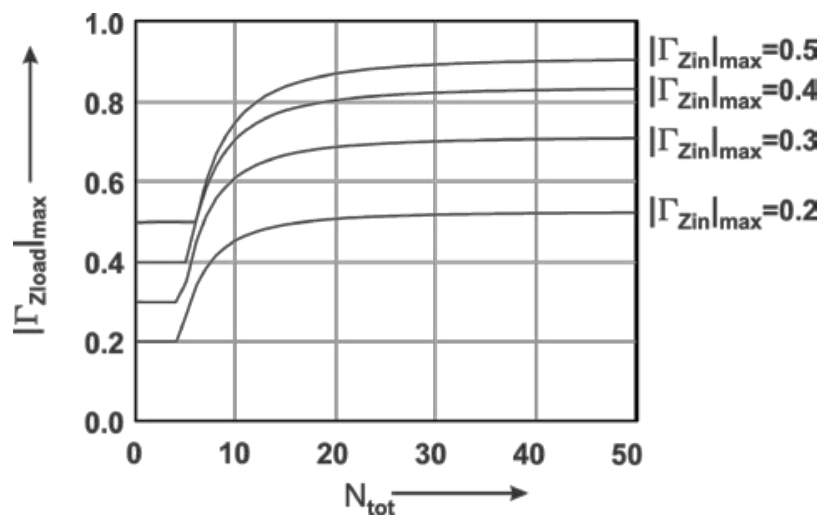

Fig. 4. Radius of the matchable region $\left|\Gamma_{\text {Zload }}\right|_{\max }$ as a function of the number of matching network states $N_{\text {tot }}$, for various $\left|\Gamma_{\text {Zin }}\right|_{\max }$.

\section{Two ring impedance matching}

The analysis in the previous section can be extended in a recursive way to get e.g. the circular region on the Smith chart that is composed of a center circle and two rings composed of circles that all can be matched to the center ring. For the two-ring case it can be derived that:

$$
\begin{aligned}
& \left|\Gamma_{\text {Zload }}\right|_{\max 2}=\Gamma_{\text {Zload max } 1}\left(\left|\Gamma_{\text {Zin }}\right|_{\text {max }}, N_{1}\right)+ \\
& 2 r_{2}\left(\left|\Gamma_{\text {Zload ,matched } 2}\right|, N_{2}\right) \cos \left(\frac{\pi}{N_{2}}+\varphi\left(\left|\Gamma_{\text {Zload, matched } 2}\right|\right)\right),
\end{aligned}
$$

where the first term on the right hand side in (8) equals (5) and with:

$$
\begin{aligned}
& \varphi(\gamma)=\arcsin \left(\frac{\gamma}{r_{2}} \sin \left(\frac{\pi}{N_{2}}\right)\right), \\
& r_{2}(x, N)=\frac{\left(1-h_{2}(x, N)^{2}\right) x}{1-h_{2}(x, N)^{2} x^{2}}, h_{2}(x, N)=\frac{2 x \cos \left(\frac{\pi}{N}\right)}{1+x^{2}}, \\
& \left|\Gamma_{\text {Zload, matched } 2}\right|=\frac{-b+\sqrt{b^{2}-4 d}}{2}, \\
& b=\frac{2\left(1-\left|\Gamma_{\text {Zin }}\right|_{\text {max }}^{2}\right)\left|\Gamma_{\text {Zload }}\right|_{\text {max } 1} \cos \left(\frac{\pi}{N_{2}}\right)}{1-\left|\Gamma_{\text {Zload }}\right|_{\text {max } 1}^{2}\left|\Gamma_{\text {Zin }}\right|_{\text {max }}^{2}}, \\
& d=\frac{\left|\Gamma_{\text {Zload }}\right|_{\max 1}^{2}-\left|\Gamma_{\text {Zin }}\right|_{\max }^{2}}{1-\left|\Gamma_{\text {Zload }}\right|_{\text {max }}^{2}\left|\Gamma_{\text {Zin }}\right|_{\max }^{2}},
\end{aligned}
$$

where again $\left|\Gamma_{\text {Zin }}\right|_{\max }$ is the required magnitude of the reflection coefficient at the input of the switchable matching network, $N_{1}$ and $N_{2}$ are the number of circles of the first and second ring respectively. Also in case of two rings some trivial boundary conditions must be satisfied to get a valid solution: e.g. $\left|\Gamma_{\text {Zload }}\right|_{\max 2}$ must be real valued and $\left|\Gamma_{\text {Zload }}\right|_{\max 2}>\left|\Gamma_{\text {Zload }}\right|_{\max 1}$. Fig. 5 shows an example of a two-ring configuration for a matching network. In this configuration, any of the rings (here $N_{1}=10, N_{2}=24$ ) can exactly be matched to the center ring, thereby being able to match any impedance $\left|\Gamma_{\text {Zload }}\right| \leq\left|\Gamma_{\text {Zload }}\right|_{\text {max } 2}$ to within $\left|\Gamma_{\text {Zin }}\right| \leq\left|\Gamma_{\text {Zin }}\right|_{\max }$. 


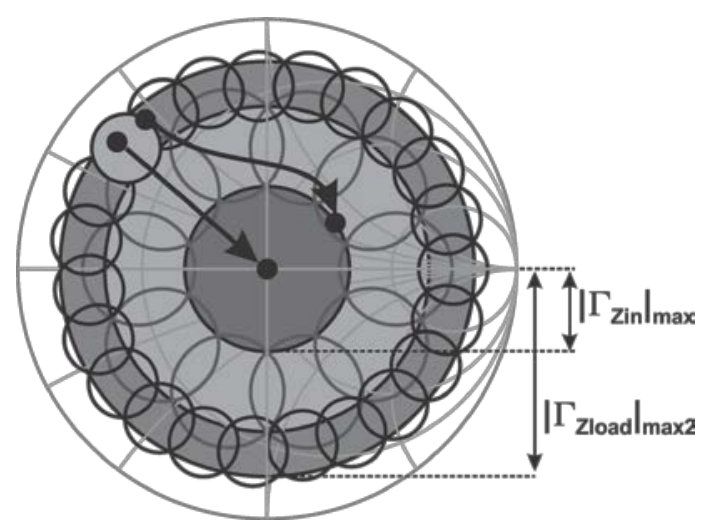

Fig. 5. Constructing a area with radius $\left|\Gamma_{\text {Zload }}\right|_{\max 2}$ matchable onto a circle around the origin with radius $\left|\Gamma_{\text {Zin }}\right|$, using multiple (here 35) number of states in a two-ring configuration.

It appears that it is at least hard to derive a closed mathematical expression for the maximum circular region on the Smith chart that can be mapped onto the center circle for the two-ring case. This is basically because an optimum in (8)-(9) for a constant number of total states $N_{\text {tot }}=\left(N_{2}+N_{1}+1\right)$ cannot readily be derived. These optima can however quite easily be derived numerically as only integer $N_{1}$ and $N_{2}$ occur.

Fig. 6 shows a contour plot the radius of the regions that can be matched as a function of $N_{1}$ and $N_{2}$, here for $\left|\Gamma_{\text {Zin }}\right|_{\max }=1 / 3$. For low $N_{\text {tot }}$ this behavior is identical to that shown in Fig. 4 for the single-ring configuration of the matching network: the maximum $\left|\Gamma_{\text {Zload }}\right|_{\max }$ occurs for $N_{2}=0$. For higher $N_{\text {tot }}$ a second ring gets to become beneficial and then the maximum $\left|\Gamma_{\text {zload }}\right|_{\max }$ is achieved for $N_{2}>0$.

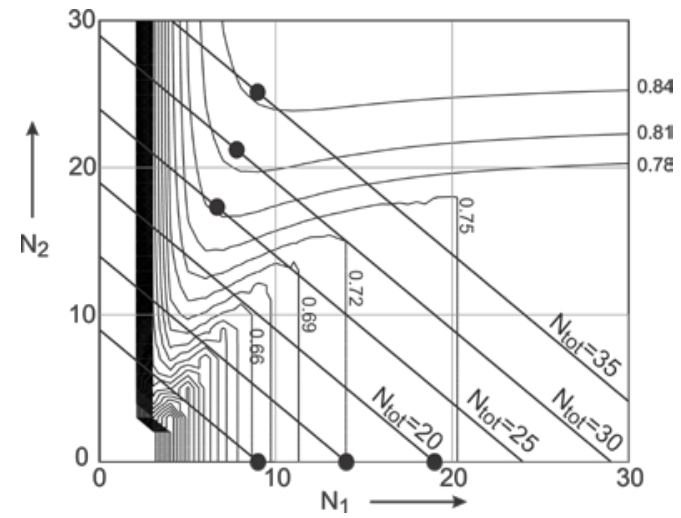

Fig. 6. Contour plot of $\left|\Gamma_{\text {Zload }}\right|_{\text {max }}$ as a function of $N_{1}$ and $N_{2}$, here for $\left|\Gamma_{\text {Zin }}\right|_{\text {max }}=1 / 3$. On the plot, constant- $N_{\text {tot }}$ lines are plotted for $N_{\text {tot }}=10$, 15, ... 35. On these lines, the maximum matchable $\left|\Gamma_{\text {Zload }}\right|_{\max }$ is denoted with a dot.

A straight forward maximum search for constant $N_{\text {tot }}=\left(N_{2}+N_{1}+1\right)$ directly yields the maximum magnitude of the load reflection coefficient $\left|\Gamma_{\text {Zload }}\right|_{\max }$ as a function of both the total number of states $N_{\text {tot }}$ and of $\left|\Gamma_{\text {Zin }}\right|_{\max }$. Fig. 7 shows both $\left|\Gamma_{\text {Zload }}\right|_{\text {max }}$ as a function of $N_{\text {tot }}$ for (here) $\left|\Gamma_{\text {Zin }}\right|_{\max }=1 / 3$ and shows the distribution of $N_{\text {tot }}$ across the first and second ring. In this example, for $N_{\text {tot }}<6$ no continuous band can be formed, while for $6 \leq N_{\text {tot }}<21$ a single configuration ring is optimum. For larger $N_{\text {tot }}$ the two-ring solution is most efficient and can achieve matching of a larger $\left|\Gamma_{\text {Zload }}\right|_{\text {max }}$ region compared to the single-ring case. 


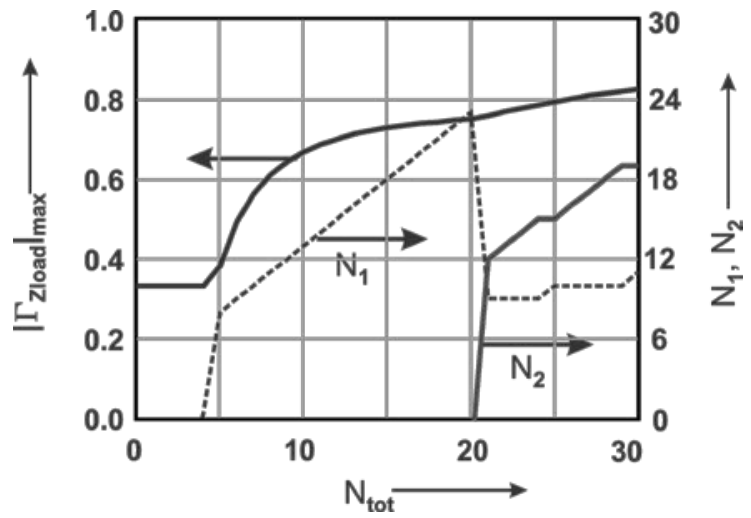

Fig. 7. Matchable region impedances on the Smith chart as a function of the total number of states ( $N_{\text {tot }}$ ), for a two-ring network, for $\left|\Gamma_{\text {Zin }}\right|_{\text {max }}=1 / 3$ (VSWR = 2:1). The plot also shows the optimum $N_{1}$ and $N_{2}$ as a function of $N_{\text {tot }}$.

\section{MUlTiSTAGE LOSSLESS SWITCHABLE MATCHING NETWORKS}

The previous section showed an analysis of single stage matching networks, that used matching states in one or two rings to match any impedance $\left|\Gamma_{\text {Zload }}\right| \leq\left|\Gamma_{\text {Zload }}\right|_{\text {max }}$ to within $\left|\Gamma_{\text {Zin }}\right|_{\max }$. It was also shown that a two-ring configuration can match a larger $\left|\Gamma_{\text {Zload }}\right|_{\max }$ than a single-ring matching network configuration. This section shows that multi-stage single-ring configurations, see Fig. 8 for a two-stage matching network, outperform single-stage multi-ring matching networks in terms of (e.g.) minimum required total number of states.

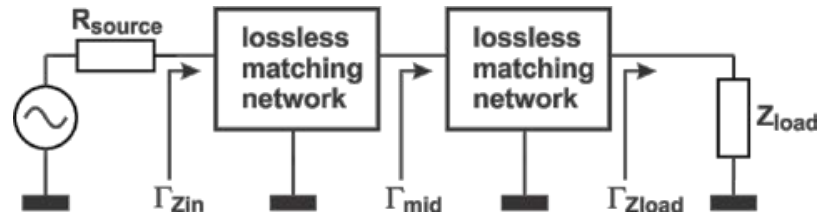

Fig. 8. Block diagram of a two-stage switchable matching network.

In multi-stage networks, the switchable matching network connected to the load achieves coarse tuning from $\Gamma_{\text {zload }}$ to $\Gamma_{\text {mid }}$ while the leftmost network achieves fine tuning from $\Gamma_{\text {mid }}$ to $\Gamma_{\text {Zin }}$; this principle is shown in Fig. 9 for matching for any impedance within $\left|\Gamma_{\text {Zload }}\right| \leq\left|\Gamma_{\text {Zload }}\right|_{\max }$ via $\Gamma_{\text {mid }}$ to within $\left|\Gamma_{\text {Zin }}\right|_{\max }$.

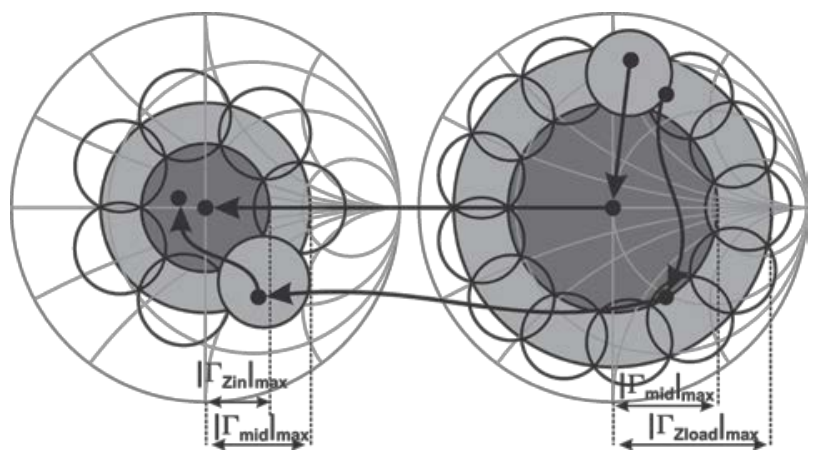

Fig. 9. Matching in a two-stage switchable matching network: the second stage (right hand side Smith chart) matches an impedance to within $\left|\Gamma_{\text {mid }}\right|_{\text {max }}$; the first stage (left hand side Smith chart) matches this to within $\left|\Gamma_{\text {Zin }}\right|_{\max }$.

It follows directly from bilinear properties, and from the analyses in section II that this approach to match a relatively large $\left|\Gamma_{\text {Zload }}\right| \leq\left|\Gamma_{\text {Zload }}\right|_{\text {max }}$ to within a circle $\left|\Gamma_{\text {Zin }}\right|_{\text {max }}$ is much more efficient than using multi-ring approaches because mainly the coarse matching stage uses relatively large circles that require a relatively low minimum number of (large) circles to cover a large part of the Smith chart. For illustration purposes, the $\left|\Gamma_{\text {Zload }}\right|_{\max }$ and $\left|\Gamma_{\text {Zin }}\right|_{\max }$ for Fig.9 are identical to the ones used for Fig. 5, requiring 
35 states in total for the single stage network and 22 in total for the multi-stage solution. This difference in minimum number of required matching network states increases rapidly for larger ratios between $\left|\Gamma_{\text {Zload }}\right|_{\text {max }}$ and $\left|\Gamma_{\text {Zin }}\right|_{\text {max }}$.

\section{A. $\left|\Gamma_{\operatorname{mid}}\right|_{\max }$ estimation}

In case of required good input matching (i.e. low $\left|\Gamma_{Z i n}\right|_{\max }$ ), a good approximation of the optimum maximum magnitude of the reflection coefficient at the intermediate cross section, $\left|\Gamma_{\operatorname{mid}}\right|_{\max }$, can be derived. Assuming a small $\left|\Gamma_{\text {Zin }}\right|_{\max }$, (5) can be simplified to $\left|\Gamma_{\text {Zload }}\right|_{\max } \cong\left|\Gamma_{\text {Zin }}\right|_{\max }\left(1+2 \cos \left(\frac{2 \pi}{N}\right)\right)$, yielding for a two-stage matching network having one ring per stage $\left|\Gamma_{\text {Zload }}\right|_{\text {max }} \cong\left|\Gamma_{\text {Zin }}\right|_{\text {max }}\left(1+2 \cos \left(\frac{2 \pi}{N_{1}}\right)\right) \cdot\left(1+2 \cos \left(\frac{2 \pi}{N_{\text {tot }}-N_{1}-2}\right)\right)$.

Due to symmetry, equal distribution of the matching network states over the two stages is optimum, yielding

$$
\begin{aligned}
\left|\Gamma_{\text {mid }}\right|_{\text {max }} & \cong\left|\Gamma_{\text {Zin }}\right|_{\text {max }}\left(1+2 \cos \left(\frac{2 \pi}{N / 2-1}\right)\right) . \\
& =\sqrt{\left|\Gamma_{\text {Zin }}\right|_{\text {max }}\left|\Gamma_{\text {Zload }}\right|_{\text {max }}}
\end{aligned}
$$

For $\left|\Gamma_{\text {Zin }}\right|_{\max }$-values that are not very small an easy-to-read expression for $\left|\Gamma_{\operatorname{mid}}\right|_{\max }$ cannot be derived. However, combining the result of two one-stage one-ring results to get multi-stage results is quite easy, with just one numerical (integer) search required to get the optimum $\left|\Gamma_{\operatorname{mid}}\right|_{\max }$.

\section{B. Benchmarking lossless multi-stage matching networks}

Fig. 10 shows 4 curves, all showing $\left|\Gamma_{\text {Zload }}\right|_{\max }$ as a function of the total number of states in the matching network $N_{\text {tot }}$, for $\left|\Gamma_{\text {Zin }}\right|_{\max }=1 / 3$. The curves are for single-stage matching networks with either one of two rings of circles optimally positioned around the center ring and are for cascaded single-stage single-ring configurations.

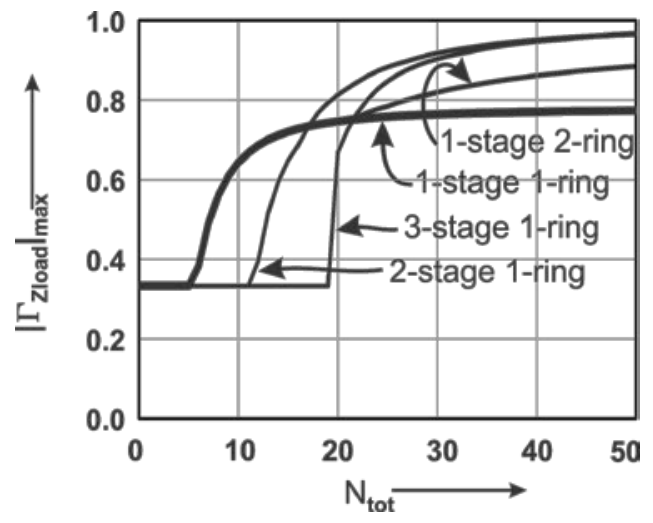

Fig. 10. Maximum magnitude of load reflection coefficient $\left(\left|\Gamma_{\text {load }}\right| \max \right)$ as a function of the total number of states ( $\left.\mathrm{N}_{\text {tot }}\right)$ for one-stage-one-ring, one-stage-tworing, two-stage-one-ring and three-stage-one-ring networks. For the plot $\left|\Gamma_{\mathrm{Zin}}\right|=1 / 3(\mathrm{VSWR}=2: 1)$.

It follows that for maximum load reflection coefficients $\left|\Gamma_{\text {Zload }}\right|_{\text {max }}$ close to the required magnitude of the input reflection coefficient $\left|\Gamma_{\text {Zin }}\right|_{\text {max }}$, it is best to use the one-stage one-ring configuration. As $\left|\Gamma_{\text {Zload }}\right|_{\max }$ (or the total number of states for the matching network) increases, after some point it is more efficient to use any of the other configurations. However, the curves show that a single-stage multi-ring configuration is not efficient: either it degenerates to a single-stage single-ring configuration for low $N_{\text {tot }}$ or it is better to use a two-stage single-ring configuration.

Moving to more stages (i.e. three-stage switchable matching networks) appears to be useful only if very large $\left|\Gamma_{\text {zload }}\right|_{\text {max }}$ must be matched onto small $\left|\Gamma_{\text {Zin }}\right|_{\max }$. In the case of adaptive antenna matching, these cases typically represent broken antenna's or electromagnetically very bad environments. In this case switching off the PA might be a better option. 


\section{COMPLEX SOURCE IMPEDANCES}

For simplicity reasons, the derivations in section II and III were done assuming a resistive source, with a straight forward extension towards generators with an arbitrary impedance $Z_{\text {source }}$ that require a conjugate $Z_{\text {in }}$ of the matching network (see Fig. 1) for maximum power transfer.

For RF power amplifiers, complex conjugate matching is typically not used but a specific load impedance is set to be able to get maximum power or maximum efficiency, determined via e.g. load pull. Whereas mismatch efficiency contours are circles on the Smith chart, load pull contours usually are not [26]; they generally address a non-linear problem. In this case the analysis shown in this paper would only give a coarse upper bound estimation on the number of required states for an adaptive matching network.

However, usually in PA design the electrical length between the PA and the actual load is unknown or unspecified which results in having to accept an arbitrary phase shift in the load impedance. This means that actual PAs usually must be designed for the smallest area enclosed by arbitrarily rotated load pull contours. This smallest enclosed area equals the largest circle enclosed by the not-rotated load pull contour. For this circular area, the analyses shown in this paper hold.

\section{LOSSY MATCHING NETWORKS}

In sections II and III, requirements on the minimum required number of states were derived for general lossless switchable impedance matching networks. In reality, switchable matching networks are lossy, although with low loss to have any significance as impedance matching network. This section presents an extension of the work in II and III towards lossy matching networks. Note that also in this section no specific hardware implementation is assumed to keep the results as general as possible.

Any two-port linear network can be described as an 2x2 S- or ABCD matrix; in this section first the ABCD-representation is used. As shown in Appendix B, the ABCD-matrix of a lossy matching network $\left\lfloor A B C D_{\text {lossy }}\right\rfloor$ can be decomposed in 3 ABCDmatrices and an extra phase shift:

$$
\begin{aligned}
{[A B C D]_{\text {lossy }}=} & {[A B C D]_{\text {attenuator } 1} \cdot[A B C D]_{\text {lossless }} . } \\
& {[A B C D]_{\text {attenuator } 2} \cdot e^{j \phi} }
\end{aligned}
$$

where $[A B C D]_{\text {attenuator }}$ are purely real lossy circuits, with in total (at least) 3 degrees of freedom, and $[A B C D]_{\text {lossless }}$ is a lossless (reciprocal or non-reciprocal) matching network having 4 degrees of freedom as assumed in sections II-V in this paper, see Fig. 11.

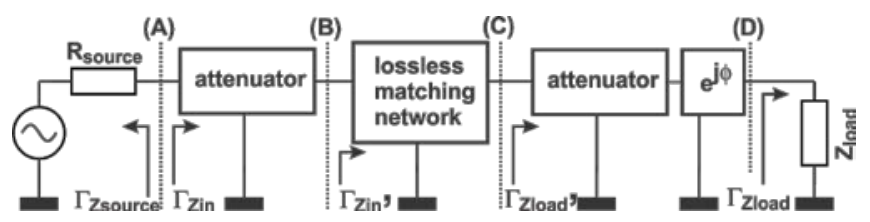

Fig. 11. Equivalent block diagram of a lossy switchable matching network: a the cascade of two attenuators, a lossless switchable matching network and a phase shifter.

In other to extend the results in sections II-V to lossy matching networks, it is useful to switch back to the S matrix. Using the decomposition of the lossy network into a lossless network with attenuators (and a phase shifter) wrapped around it, the effect of losses can be illustrated and calculated. For the illustrations, relatively high losses are used for visibility reasons.

In Fig. 11 the lossless network is between planes (B) and (C). For this part of the lossy matching network, the analyses in section II-III hold. An example of the corresponding matchable region and circle to match to are shown in Fig. 12C and Fig. 12B respectively.

The effect of an attenuator is twofold. Firstly the attenuation scales up the radius of the matchable circle on the Smith chart when moving towards the load side of the matching network. Assuming only losses in the attenuator, so having $S_{11, n}=S_{22, n}=0$ and having a lower-than-unity magnitude of $S_{21, n}$ for the $n^{\text {th }}$ attenuator, it follows that the center point does not shift and $\left|\Gamma_{\text {Zin }}\right|_{\max }=\left|S_{21,1}\right|^{2}\left|\Gamma_{\text {Zin }}\right|_{\text {max }}$. Secondly, for a more general attenuator $S_{11, n} \neq S_{22, n}$, which results in a change of both the radius and the center point of the circles according to $\Gamma_{\text {Zin }}=S_{11,1}+\frac{S_{21,1} S_{12,1} \Gamma_{Z^{\prime} n^{\prime}}}{1-S_{22,1} \Gamma_{Z^{\prime} n^{\prime}}}$.

The power efficiency of the lossy matching network is 


$$
P_{\text {load }}=\left|S_{21,1}\right|^{2} \frac{1-\left|\Gamma_{\text {Zin }}\right|^{2}}{1-\left|\Gamma_{\text {Zin }}\right|^{2}}\left|S_{21,2}\right|^{2} \frac{1-\left|\Gamma_{\text {Zload }}\right|^{2}}{1-\left|\Gamma_{\text {Zlood }}\right|^{2}} P_{\text {in }}
$$

where $\mathrm{P}_{\text {load }}$ is the power to the load, $\mathrm{P}_{\text {in }}$ is the input power and the other symbols are defined in figure 11.

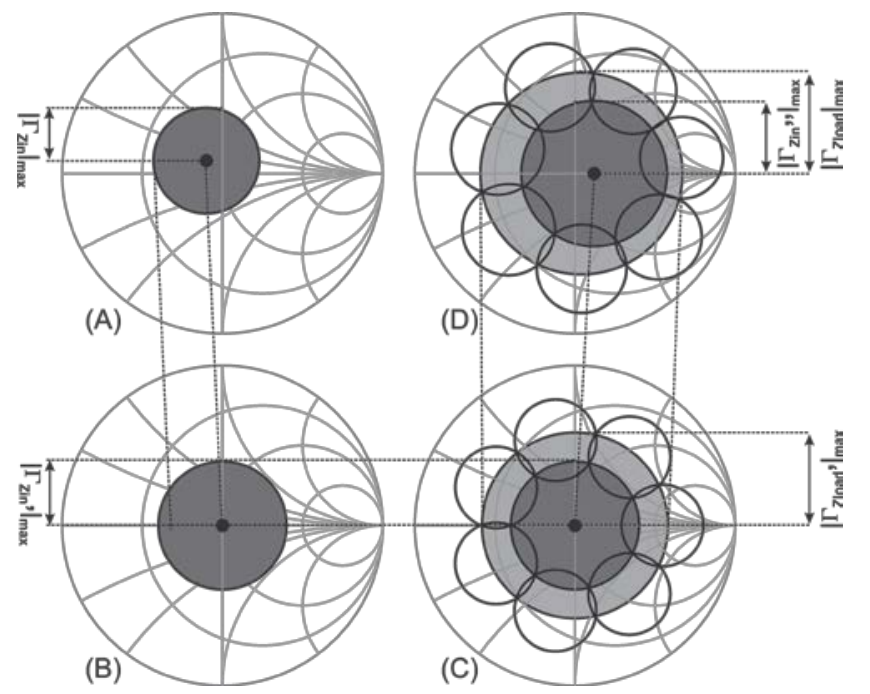

Fig. 12. Transformation of the Smith chart moving from the input to the output: A) input impedance region of the lossy network: in order to make the plot

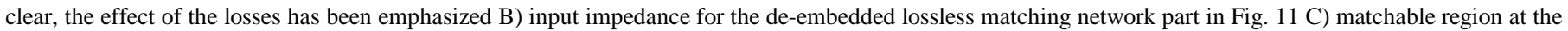

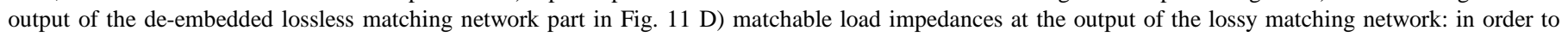
make the plot clear, the effect of the losses has been emphasized.

\section{CONCLUSION}

This paper shows a mathematical derivation for the minimum number of states for passive lossless switchable matching networks to match any load impedance for which $\left|\Gamma_{\text {Zload }}\right|<\left|\Gamma_{\text {Zload }}\right|_{\max }$ to an impedance for which $\left|\Gamma_{\text {Zin }}\right|<\left|\Gamma_{\text {Zin }}\right|_{\max }<\left|\Gamma_{\text {Zload }}\right|_{\max }$. In this paper, the switchable matching network can be reciprocal or non-reciprocal.

For relatively simple single-stage matching networks, a closed form expression is derived for the largest matchable region $\left|\Gamma_{\text {zload }}\right|<\left|\Gamma_{\text {zload }}\right|_{\max }$ as a function of maximum input reflection $\left|\Gamma_{\text {zin }}\right|_{\max }$ and of the number of states for the matching network.

Several other configurations were studied: more complex one stage configuration as well as cascaded simple matching networks. It appears that at low ratios between $\left|\Gamma_{\text {Zin }}\right|_{\text {max }}$ and $\left|\Gamma_{\text {Zload }}\right|_{\text {max }}$ the one-stage one-ring configuration is the best choice. As the mismatch between required input impedance and required load impedance increases, a cascaded configuration consisting of simple matching networks becomes the best option. The analysis has been extended to lossy passive switchable matching networks; the main difference with the lossless situation is a shift and scaling of the matchable region.

\section{APPENDIX A}

This appendix presents the mathematical derivation of matchable region in case of a single-ring switchable matching networks, described by $\left|\Gamma_{\text {Zload }}\right|_{\max }$ in (5) and (6). The region that can be matched using a single-ring containing $N_{1}$ evenly spaced circles that individually can be matched onto the center ring can be derived by geometrical construction under constrains imposed by bilinear transformations and e.g. (2).

From a geometrical point-of-view, see Fig. 13 for an example configuration that shows just the center circle and 2 circles in the first ring, the distance $c$ between the origin of the Smith chart and the center of one of the $N_{1}$ circles in the ring is given by

$$
c=\left|\Gamma_{\text {zin }}\right|_{\max } \cos (\alpha)+r \cos (\beta),
$$

where the angle $\alpha$ directly follows from the equidistant spacing, $\alpha=\pi / N_{1}$. The radius of the smaller circles $r$ and the angle $\beta$ are related as $r \cdot \sin (\beta)=\left|\Gamma_{\text {Zin }}\right|_{\max } \sin \left(\pi / N_{1}\right)$. Using this, the region that can be matched to within the inner circle is a circle centered on the origin of the Smith chart, having a radius 
$\left|\Gamma_{\text {Zload }}\right|_{\max }=\left|\Gamma_{\text {Zin }}\right|_{\max }+2 r \cos \left(\frac{\pi}{N_{1}}+\beta\right)$.

From a bilinear point-of-view, (2) can be used to get another set of equations for $r$ and $c$ that also must be satisfied:

$$
r=\frac{\left.\left(1-\left|\Gamma_{\text {Zload }}\right|_{\text {max }}^{2}\right) \Gamma_{\text {Zin }}\right|_{\text {max }}}{1-\left|\Gamma_{\text {Zload }}\right|_{\text {max }}^{2}\left|\Gamma_{\text {Zin }}\right|_{\text {max }}^{2}},
$$

and

$$
c=\frac{\left|\Gamma_{\text {Zload }}\right|_{\text {max }}\left(1-\left|\Gamma_{\text {Zin }}\right|_{\text {max }}^{2}\right)}{1-\left|\Gamma_{\text {Zload }}\right|_{\text {max }}^{2}\left|\Gamma_{\text {Zin }}\right|_{\text {max }}^{2}},
$$

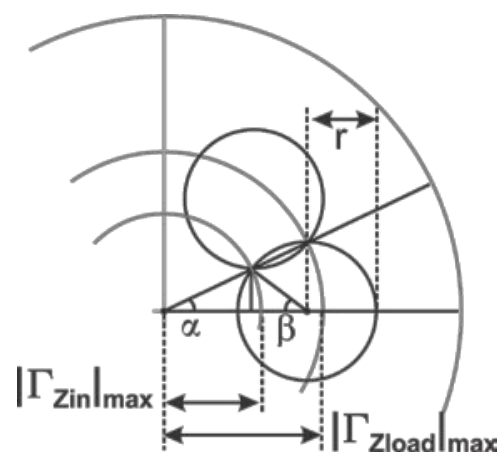

Fig. 13. Geometric construction to derive the matchable region for one-stage one-ring switchable matching networks.

Substituting (13)-(14) into (12) yields

$$
\begin{aligned}
& \frac{\left|\Gamma_{\text {Zload }}\right|_{\text {max }}\left(1-\left|\Gamma_{\text {Zin }}\right|_{\text {max }}^{2}\right)}{1-\left|\Gamma_{\text {Zload }}\right|_{\text {max }}^{2}\left|\Gamma_{\text {Zin }}\right|_{\text {max }}^{2}}=\left|\Gamma_{\text {in }}\right| \cos \left(\frac{\pi}{N_{1}}\right)+ \\
& \frac{\left.\left(1-\left|\Gamma_{\text {Zload }}\right|_{\text {max }}^{2}\right) \Gamma_{\text {Zin }}\right|_{\text {max }}}{1-\left|\Gamma_{\text {Zload }}\right|_{\text {max }}^{2}\left|\Gamma_{\text {Zin }}\right|_{\text {max }}^{2}} \sqrt{1-\frac{\left(1-\left|\Gamma_{\text {Zload }}\right|_{\text {max }}^{2}\left|\Gamma_{\text {Zin }}\right|_{\text {max }}^{2}\right)^{2}}{\left(1-\left|\Gamma_{\text {Zload }}\right|_{\text {max }}^{2}\right)^{2}} \sin ^{2}\left(\frac{\pi}{N_{1}}\right)}
\end{aligned}
$$

Eliminating the square root and using $\sin ^{2}(\mathrm{x})+\cos ^{2}(\mathrm{x})=1$, the previous equation can be rewritten into an equation that is $6^{\text {th }}$ order in $\left|\Gamma_{\text {Zin }}\right|_{\text {max }}$ and $4^{\text {th }}$ order in $\left|\Gamma_{\text {Zload }}\right|_{\max }$. This equation can be rewritten into an equation that is $4^{\text {th }}$ order in $\left|\Gamma_{\text {Zin }}\right|_{\text {max }}$ and $3^{\text {rd }}$ order in $\left|\Gamma_{\text {zload }}\right|_{\max }$ under the (always satisfied) constraints that $\left|\Gamma_{\text {Zin }}\right|_{\max } \neq 1$ and $\left|\Gamma_{\text {Zload }}\right|_{\max } \neq 0$ :

$$
\begin{aligned}
& \left(1-\left|\Gamma_{\text {Zin }}\right|_{\text {max }}^{2}\left|\Gamma_{\text {Zload }}\right|_{\text {max }}^{2}\right) * \\
& *\left[\left.\left(1+\left|\Gamma_{\text {Zin }}\right|_{\text {max }}^{2}\right) \Gamma_{\text {Zload }}\right|_{\text {max }}-2\left|\Gamma_{\text {in }}\right|_{\text {max }} \cos \left(\frac{\pi}{N_{1}}\right)\right]=0 .
\end{aligned}
$$

Excluding a trivial (and for matching networks invalid) solution $\left|\Gamma_{\text {Zload }}\right|_{\max }=\left|\Gamma_{\text {Zin }}\right|_{\max }^{-1}$, this directly yields

which leads to

$$
\left.\left(1+\left|\Gamma_{\text {in }}\right|_{\text {max }}^{2}\right) \Gamma_{\text {zload }}\right|_{\max }-2\left|\Gamma_{\text {in }}\right|_{\text {max }} \cos \left(\frac{\pi}{N_{1}}\right)=0,
$$

$$
\left|\Gamma_{\text {Zload }}\right|_{\text {max }}=\frac{2\left|\Gamma_{\text {in }}\right|_{\text {max }} \cos \left(\frac{\pi}{N_{1}}\right)}{1+\left|\Gamma_{\text {in }}\right|_{\text {max }}^{2}}
$$

with (1) and (4), (15) yields (5) and (6). 


\section{APPENDIX B}

This appendix shows some background on the decomposition of the transfer function of an arbitrary lossy matching network into a cascade of transfer functions, one of which corresponding to the transfer of a lossless matching network [27-29]. Any two-port linear network can be described as an 2x2 S- or ABCD matrix. For a lossless network, the on-diagonal elements in the ABCDmatrix are real and the off-diagonal elements are imaginary. For lossy networks the power gain is lower than unity which results in additional constraints which are irrelevant for the decomposition in this appendix. In general the ABCD-matrix of any lossless matching network therefore has 4 degrees of freedom. For reciprocal circuits, $\operatorname{det}(A B C D) \equiv|A B C D|=1$ and the number of degrees of freedom would be 3 ; however in this paper no reciprocity is assumed.

The transfer of an arbitrary (lossy) network can be written in a complex 2x2 ABCD-matrix with no constraints on any element in that matrix: the ABCD-matrix then has 8 degrees of freedom. This general ABCD-matrix can be decomposed into multiple ABCD-matrices (one of which corresponds to a lossless network) in a number of ways. Common to all these decompositions is that the total number of degrees of freedom is (at least) 8 and that the decomposition can be made. This last requirement can be reformulated into being able to cover the 8 ( 4 real and 4 imaginary) dimensional space spanned by the original ABCD-matrix by the cascade of ABCD-matrices in the decomposition.

The hardest decomposition is that of the arbitrary ABCD-matrix into a cascade of ABCD-matrices with in total 8 degrees of freedom. In case that the cascade of ABCD-matrices has more degrees of freedom clearly the mapping can be made more easily. In the derivation below purely resistive attenuator circuits are assumed for simplicity reasons; equivalent circuits including transformers also may be used. Then, starting from basic mathematical rules on determinants:

$$
\begin{aligned}
& \left|A B C D_{\text {general }}\right|=\text { complex } \\
& \left|A B C D_{\text {lossless }}\right|=\text { real } \\
& \left|A B C D_{\text {attenuator }}\right|=1 \\
& \left|A B C D_{\text {phaseshifter }}\right|=\text { complex } \\
& |A| \cdot|B| \cdots|Z|=|A \cdot B \cdots Z|
\end{aligned}
$$

it directly follows that decomposing a general (lossy) ABCD-matrix $\left[A B C D_{\text {general }}\right\rfloor$ into a cascade including one lossless matching network $\left[A B C D_{\text {lossless }}\right]$ requires at least a phaseshifter $\left\lfloor A B C D_{\text {phaseshifter }}\right\rfloor$ and 2 lossy attenuators $\left[A B C D_{\text {attenuator }}\right]$. For a decomposition into a cascade that has 8 degrees of freedom we then get e.g.

$$
\begin{aligned}
{[A B C D]_{\text {general }}=} & {[A B C D]_{\text {attenuator } 1} \cdot[A B C D]_{\text {lossless }} \text {. } } \\
& {[A B C D]_{\text {attenuator } 2} \cdot e^{j \phi} }
\end{aligned}
$$

Using the inverse function theorem it can be shown that this decomposition can be made with attenuators having in total 3 degrees of freedom (total insertion loss and impedance ratios). Using more degrees of freedom in the attenuators enables infinitely many ways to decompose the general transfer function in the way shown in the above relation.

\section{REFERENCES}

[1] K. Boyle, M. Leitner, "Mobile phone antenna impedance variations with real users and phantoms", 2011 International Workshop on Antenna Technology (iWAT), pp. 420 - 423.

[2] E.L. Firrao, A.J. Annema, B. Nauta, "Antenna behaviour in the presence of human body", ProRISC 2004, 15th Annual Workshop on Circuits, Systems and Signal Processing, 25 - 26 Nov 2004.

[3] A. Scuderi, L. La Paglia, A. Scuderi, F. Carrara, G. Palmisano, “A VSWR-Protected Silicon Bipolar RF Power Amplifier With Soft-Slope Power Control”, IEEE J. Solid-State Circuits, Vol. 40, No. 3, pp. 611 - 621, March 2005.

[4] F. Carrara, C. D. Presti, A. Scuderi, C. Santagati, G. Palmisano, "A Methodology for Fast VSWR Protection Implemented in a Monolithic 3-W 55\% PAE RF CMOS Power Amplifier”, IEEE J. Solid-State Circuits, Vol. 43, No. 9, pp. 2057 - 2066, September 2008.

[5] A. van Bezooijen, R. Mahmoudi, A. H. M. van Roermund, "Adaptive Methods to Preserve Power Amplifier Linearity Under Antenna Mismatch Conditions”, IEEE Trans. on Circuits Syst. - I, Reg. Papers, Vol. 52, No 10, pp. 2101 - 2108, October 2005.

[6] J. de Mingo, A. Valdovinos, A. Crespo, D. Navarro, P. García, “An RF Electronically Controlled Impedance Tuning Network Design and Its Application to an Antenna Input Impedance Automatic Matching System”, IEEE Trans. Microw. Theory Techn., Vol 52, No 2, pp. 489 - 497, February 2004.

[7] C. Hoarau, N. Corrao, J.-D. Arnould, P. Ferrari, P. Xavier, "Complete Design and Measurement Methodology for a Tunable RF Impedance-Matching Network”, IEEE Trans. Microw. Theory Techn., Vol 56, No 11, pp. 2620 - 2627, November 2008.

[8] F. Domingue, S. Fouladi, A. B. Kouki, R. R. Mansour, "Design Methodology and Optimization of Distributed MEMS Matching Networks for LowMicrowave-Frequency Applications”, IEEE Trans. Microw. Theory Techn., Vol 57, No 12, pp. 3030 - 3041, December 2009.

[9] Q. Gu, A. S. Morris III, “A New Method for Matching Network Adaptive Control”, IEEE Trans. Microw. Theory Techn., Vol 61, No 1, pp. 587 - 595, January 2013.

[10] C. Sánchez-Pérez, J. de Mingo, P. L. Carro, P. García-Dúcar, “Design and Applications of a 300-800 MHz Tunable Matching Network”, IEEE J. Emerg. Sel. Topic Circuits Syst., Vol 3, No 4, pp. 531 - 540, December 2013.

[11] H. Song, J. T. Aberle, B. Bakkaloglu,” A Mixed-Signal Matching State Search Based Adaptive Antenna Tuning IC”, IEEE Microw. Wireless Compon. Lett., Vol. 20, No. 10, pp. 581 - 583, October 2010.

[12] A. van Bezooijen, M. A. de Jongh, C. Chanlo, L. C. H. Ruijs, F. van Straten, R. Mahmoudi, A. H. M. van Roermund, "A GSM / EDGE / WCDMA Adaptive Series-LC Matching Network Using RF-MEMS Switches”, IEEE J. Solid-State Circuits, Vol. 43, No. 10, pp. 2259 - 2268 , October 2008. 
[13] Q. Gu, J. R. De Luis, A. S. Morris III, J. Hilbert, “An Analytical Algorithm for Pi-Network Impedance Tuners”, ”, IEEE Trans. on Circuits Syst. - I, Reg. Papers, Vol. 58, No 12, pp. 2894 - 2905, December 2011.

[14] E.L. Firrao, A.J. Annema, B. Nauta, "An automatic antenna tuning system using only RF-Signal amplitudes", IEEE Trans. on Circuits Syst. - II, Exp. Briefs, vol. 55 (9), pp 833 - 837, September 2008.

[15] K. Boyle, T. Bakker, M. de Jong, A. van Bezooijen, "A low cost dual band adaptive antenna tuning module for mobile phones", 2010 International Workshop on Antenna Technology (iWAT), pp. 1 - 4.

[16] F. Chan Wai Po, E. de Foucauld, D. Morche, P. Vincent, E. Kerherve', "A Novel Method for Synthesizing an Automatic Matching Network and Its Control Unit", IEEE Trans. on Circuits Syst. - I, Reg. Papers, Vol. 58(9), pp. 2225 - 2236, September 2011.

[17] A. van Bezooijen, M. A. de Jongh, F. van Straten, R. Mahmoudi, A. H. M. Van Roermund, "Adaptive Impedance Matching Techniques for Controlling L Networks", IEEE Trans. on Circuits Syst. - I, Reg. Papers, Vol. 57(2), pp. 495 - 505, February 2010.

[18] Y. Yoon, H. Kim, K. Chae, J. Cha, H. Kim, C.H. Lee, “An Antenna Mismatch Immuned CMOS Power Amplifier”, IEEE Solid-State Circuits Conference, November 8-10 2010, Beijing, China, pp. 1 - 4.

[19] F. Sonnerat, R. Pilard, F. Gianesello, F. Le Pennec, C Person, D. Gloria, "4G Antenna Tuner Integrated in a 130 nm CMOS SOI Technology", IEEE 12th Topical Meeting on Silicon Monolithic Integrated Circuits in RF Systems (SiRF), 2012, pp. 191 - 194.

[20] A. M. M. Mohamed, S. Boumaiza, R. R. Mansour, "Novel Reconfigurable Fundamental Harmonic Matching Network for Enhancing the Efficiency of Power Amplifiers", Proceedings of the 40th European Microwave Conference, 2010 , pp. 1122 - 1125.

[21] www.sgcworld.com

[22] K. B. de Brito, R. N. de Lima, "Impedance Network for an Automatic Impedance Matching System", Proceedings of Asia Pacific Microwave Conference 2007, pp. 1 - 4 .

[23] K. Brito, R. N. de Lima, "Tunable Impedance Matching Network", Microwave and Optoelectronics Conference, 2007. IMOC 2007. SBMO/IEEE MTT-S International, pp. 117 - 121.

[24] Y. de Medeiros, R. N. de Lima, F. R. de Sousa, "RF Amplifier with Automatic Impedance Matching System”, 2010 International Workshop on Circuits and Systems (LASCAS), 2011, pp. 1 - 4.

[25] R. E. Collin, Foundations for Microwave Engineering, Second Edition, McGraw Hill, 2001.

[26] S.C. Cripps, RF Power Amplifiers for Wireless Communications, Artech House, 1999.

[27] D. M. Pozar, Microwave Engineering, 4th ed. Wiley, 2011

[28] H.A. Haus, "Equivalent Circuit for a Passive Nonreciprocal Network", J. Applied Physics, vol. 25, 1954, pp 1500-1502.

[29] L.B. Felsen and A.A. Oliner, "Determination of Equivalent Circuit Parameters for Dissipative Microwave Structures”, proc. IRE, pp. 477-483, 1954.

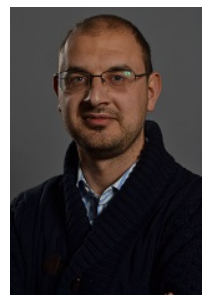

Ettore Lorenzo Firrao received the M.S. degree in electronic engineering from the University of Firenze, Firenze, Italy, in 2001.

He is currently a researcher with the ICD group, Faculty of Electrical Engineering, Mathematics and Computer Science, University of Twente, Enschede, The Netherlands. His research interests are linear circuits at RF and microwave frequencies.

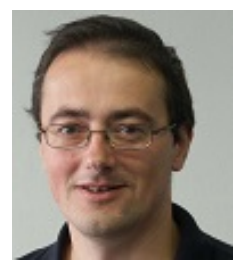

Anne-Johan Annema received the M.Sc. degree in electrical engineering and the Ph.D. degree from the University of Twente, Enschede, The Netherlands, in 1990 and 1994, respectively. In 1995, he joined the Semiconductor Device Architecture Department of Philips Research in Eindhoven, The Netherlands, where he worked on a number of physics-electronics-related projects. In 1997, he joined the Mixed-Signal Circuits and Systems Department at Philips NatLab, where he worked on a number of electronics-physics-related projects ranging from low-power low-voltage circuits, fundamental limits on analog circuits related to with process technologies, high-voltage in baseline CMOS to feasibility research of future CMOS processes for analog circuits. Since 2000 he is with the IC-Design group in the department of Electrical Engineering at the University of Twente, Enschede, The Netherlands. His current research interest is in physics, analog and mixed-signal electronics, and deepsubmicrometer technologies and their joint feasibility aspects. He is also part-time consultant in industry, co-founded ChipDesignWorks and is the recipient of four educational award at the University of Twente.

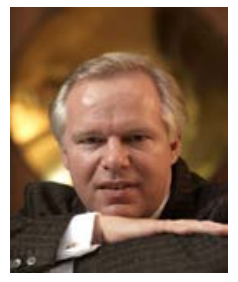

Frank van Vliet was born in Dubbeldam, The Netherlands, in 1969. He received the M.Sc. degree, with honours, in Electrical Engineering in 1992 from Delft University of Technology, The Netherlands.

Subsequently, he received his Ph.D. from the same university on MMIC filters.

He joined TNO (Netherlands Organisation for Applied Scientific Research) in 1997, where he is currently working as a principal scientist responsible for MMIC, antenna and transmit/receive module research. In 2007, he was appointed professor in microwave integration in the Integrated Circuit Design (ICD) group of the University of Twente, where he founded the Centre for Array Technology (CAT). His research interests include MMIC's in all their aspects, advanced measurement techniques and phased-array technology.

Frank van Vliet (co-)authored well over 100 peer-reviewed publications. He is a member of the European Space Agencies (ESA) Component Technology Board (CTB) for microwave components, a member of the European Defence Agencies (EDA) CapTech IAP-01, chair of the 2012 European Microwave Integrated Circuit conference (EuMIC 2012), founded the Doctoral School of 
Microwaves, and serves on the TPC of EuMIC, the IEEE International Symposium on Phased Array Systems and Technology, the IEEE Compound Semiconductor IC Symposium (IEEE CSICS) and the IEEE Conference on Microwaves, Communications, Antennas and Electronic Systems (IEEE COMCAS). He is guest editor of IEEE MTT 2013 Special issue on Phased-Array Technology.

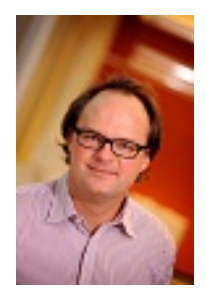

Bram Nauta was born in Hengelo, The Netherlands. In 1987 he received the M.Sc degree (cum laude) in electrical engineering from the University of Twente, Enschede, The Netherlands. In 1991 he received the Ph.D. degree from the same university on the subject of analog CMOS filters for very high frequencies. In 1991 he joined the Mixed-Signal Circuits and Systems Department of Philips Research, Eindhoven the Netherlands. In 1998 he returned to the University of Twente, as full professor heading the IC Design group. His current research interest is high-speed analog CMOS circuits.

He served as Associate Editor of IEEE Transactions on Circuits and Systems II (1997-1999). He was Guest Editor, Associate Editor (2001-2006) and later the Editor-in-Chief (2007-2010) of IEEE Journal of Solid-State Circuits. He was member of the technical program committee of the International Solid State Circuits Conference (ISSCC) where he served in several roles including the European Chair and the 2013 Program Chair. He also served in the Technical Program Committee of the Symposium on VLSI circuits (2009-2013) and is in the steering committee and programme commitee of the European Solid State Circuit Conference (ESSCIRC). He is co-recipient of the ISSCC 2002 and 2009 "Van Vessem Outstanding Paper Award". He served as distinguished lecturer of the IEEE, is member of IEEE-SSCS AdCom and is IEEE fellow. 